\title{
Conhecimento e prática assistencial dos fisioterapeutas em unidades de terapia intensiva pediátrica sobre mobilização precoce: estudo transversal
}

\section{Knowledge and care practice of physical therapists in pediatric intensive care units on early mobilization: cross-sectional study}

\author{
Emanoele Rabêlo de Brito' \\ Tatiane Falcão dos Santos Albergaria² (1) \\ Michelli Christina Magalhães Novais ${ }^{3}$ (1)
}

\author{
Maria Carolina de Britto Andrade 4 (1) \\ Tainá de Oliveira Pereira 5
}

\footnotetext{
${ }^{1}$ Autora para correspondência. Centro Universitário Jorge Amado (Salvador). Bahia, Brasil. emanoele.rabelo@gmail.com 3,5Centro Universitário Jorge Amado (Salvador). Bahia, Brasil. novaismichelli@outlook.com, tay_ellem15@hotmail.com
} 2,4Universidade do Estado da Bahia (Salvador). Bahia, Brasil. tfsalbergaria@uneb.br, mcbandrade@uneb.br

\begin{abstract}
RESUMO | INTRODUÇÃO: A mobilização precoce (MP) pode ser definida como atividade física suficiente para promover melhoras fisiológicas e redução no seu período de internação; entretanto, a prática com crianças ainda é um desafio. OBJETIVO: Avaliar o conhecimento dos fisioterapeutas atuantes em unidades de terapia intensiva pediátrica (UTIP), bem como verificar a prática clínica quanto à MP em pacientes pediátricos críticos. MATERIAL E MÉTODOS: Estudo observacional, transversal e descritivo, através de um questionário on-line. As variáveis estudadas nesta pesquisa foram referentes a três domínios, perfil e conhecimento do profissional sobre as evidências científicas existentes acerca dos benefícios da MP e percepção do participante sobre a importância da MP nestes pacientes e as barreiras vivenciadas por eles. RESULTADOS: Foram consideradas 42 respostas na coleta de resultados, $92,9 \%$ do sexo feminino, $45,2 \%$ tinham mais de 10 anos de formação em fisioterapia, e 42,8\% atuam de 5 a 10 anos em UTI pediátrica. Sobre a prática da mobilização precoce, $88,1 \%$ disseram acreditar que os estudos sugerem benefícios e 7,1\% que não há evidência científica que suporte sua realização. Todos os profissionais relataram utilizar a MP em sua rotina assistencial. CONCLUSÃO: Pode-se observar que, nesta amostra, todos profissionais realizam a prática da MP em sua rotina assistencial e que a falta de conhecimento da equipe multidisciplinar é considerada como principal barreira para realização.
\end{abstract}

PALAVRAS-CHAVE: Cuidados críticos. Unidades de Terapia Intensiva Pediátrica. Exercício. Deambulação precoce.

\begin{abstract}
INTRODUCTION: Early mobilization (MP) can be defined as sufficient physical activity to promote physiological improvements and reduce your hospital stay. Practice with children is still a challenge. OBJECTIVE: To evaluate the knowledge of physical therapists working in pediatric intensive care units (PICUs), as well as to verify the clinical practice regarding PM in critical pediatric patients. MATERIAL AND METHODS: Observational, transversal, and descriptive survey study, through an online questionnaire. The variables studied in this research were related to three domains, professional profile, professional knowledge about the existing scientific evidence about the benefits of PM, and the research participant's perception of the importance of PM in these patients and the barriers experienced by him. RESULTS: 42 responses were considered in the collection of results; $92.9 \%$ were female, $45.2 \%$ had more than 10 years of training in physical therapy, and $42.8 \%$ worked for 5 to 10 years in a pediatric ICU. Regarding the practice of early mobilization, $88.1 \%$ said they believe that the studies suggest benefits, and $7.1 \%$ that there is no scientific evidence to support its performance. All professionals reported using PM in their care routine. CONCLUSION: It can be seen that, in this observed sample, all professionals perform the practice of PM in their care routine and that the lack of knowledge of the multidisciplinary team is considered as the main barrier to performance.
\end{abstract}

KEYWORDS: Critical care. Pediatric Intensive Care Units. Exercise. Early walking.

Como citar este artigo: Brito ER, Albergaria TFS, Novais MCM, Andrade MCB, Pereira TO. Conhecimento e prática assistencial dos fisioterapeutas em unidades de terapia intensiva pediátrica sobre mobilização precoce: estudo transversal. Rev Pesqui Fisioter. 2021;11(4):647-656. http://dx.doi.org/10.17267/2238-2704rpf. v11i4.3918 


\section{Introdução}

Os efeitos deletérios funcionais adquiridos devido à imobilidade e o repouso absoluto ao leito na Unidade de Terapia Intensiva (UTI) são comuns tanto nas unidades adultos como nas pediátricas. ${ }^{1}$ Pacientes restritos ao leito por um longo período, submetidos à ventilação mecânica (VM), podem apresentar redução da força muscular periférica e respiratória, aumentando seu tempo de internação, prolongando o desmame da VM e promovendo dependência funcional. ${ }^{1}$ Durante muitos anos, era utilizado a imobilidade ao leito nos pacientes críticos, quando confinados por um longo período na UTI, por serem considerados graves para participar de terapias de mobilização.? Foram realizados estudos a partir de 1950, que comprovaram que a imobilidade era prejudicial à saúde e aos sistemas orgânicos.

A fraqueza muscular está associada a diversos fatores de risco como: imobilidade ao leito, o uso de corticoides, hiperglicemia, sepse, disfunção múltipla de órgãos, diagnóstico de Síndrome do Desconforto Respiratório Agudo (SDRA) e uso de bloqueadores neuromusculares. ${ }^{1}$ Após 48 horas de imobilidade, lesão ou doença tende-se à diminuição da força muscular, maior entre as primeiras duas a três semanas de permanência na UTI, com perda de cerca de $40 \%$ da força muscular na primeira semana de imobilização. A fraqueza muscular adquirida é relativa à idade do paciente; estudos mostram que atinge cerca de $5,1 \%$ das crianças maiores e $0,7 \%$ em crianças menores. ${ }^{4}$ Novas tecnologias e alternativas vêm sendo estudadas para minimizar os efeitos nocivos do repouso prolongado, visto que a reabilitação no início da doença crítica pode influenciar positivamente na recuperação da criança. .5

A mobilização precoce (MP) pode ser definida como movimentação física suficiente para provocar melhoras fisiológicas no paciente crítico, é realizada dentro da UTI nos primeiros dois a cinco dias da doença crítica, imediatamente após a estabilização do paciente. ${ }^{4}$ A maioria dos estudos é realizada com pacientes adultos e indicam seus benefícios. Sua prática com crianças ainda é um desafio, porém evidências recentes demonstram que a prática é viável e segura. ${ }^{6}$
Na população pediátrica, algumas atividades são descritas para a realização da mobilização precoce, que vão desde atividades de sedestação na beira do leito até deambulação e atividades específicas para idade frente à variação do desenvolvimento neurocogntivo encontrado na pediatria.?

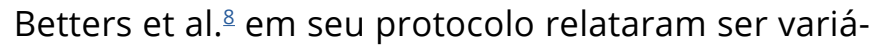
vel a duração das intervenções, tendo como referência a tolerância do paciente. A evolução do protocolo de MP é realizada através do aumento da força muscular e da coordenação do paciente, e uma vez percebida a evolução do paciente, pode ser realizada a transição dos exercícios propostos para cada vez maior independência, respeitando a idade e a fase do desenvolvimento da criança, com o objetivo de chegar à deambulação.

A ausência de diretrizes práticas específicas para população pediátrica, a falta de conhecimento da equipe multidisciplinar, a preocupação com a segurança do paciente, o nível de sedação e a disponibilidade de profissionais e recursos constituem importantes barreiras para a prática da mobilização precoce nas UTI pediátricas. 2 Desta forma, identificar como tem sido a realização da MP na prática assistencial é fundamental, o objetivo da pesquisa é avaliar o conhecimento dos fisioterapeutas atuantes em unidades de terapia intensiva pediátrica e verificar a prática clínica quanto à mobilização precoce em pacientes pediátricos críticos.

\section{Material e Métodos}

Trata-se de um estudo observacional, transversal e descritivo, o levantamento através de questionário on-line. Este estudo, de registro CAAE: 29391920.8.0000.5028 foi aprovado pelo Comitê de Ética em Pesquisa do Hospital Roberto Santos, sob o parecer de número 3.922.440, em acordo com a resolução 466/12, do Conselho Nacional de Ética em Pesquisa envolvendo seres humanos. 
Foram incluídos fisioterapeutas que atuam em UTI Pediátrica da cidade do Salvador/BA e Feira de Santana/BA. O período de coleta foi de março de 2020 a novembro de 2020, através de convite enviado por e-mail para os profissionais, com o link de acesso ao questionário da pesquisa no Google Forms, além de uma ampla divulgação realizada em grupos de mensagem de profissionais da área. Ao abrir o link, o profissional foi direcionado imediatamente para a página do questionário, onde houve através de texto o esclarecimento dos objetivos e importância da pesquisa, além do TCLE. Só foi possível o acesso do profissional às perguntas do questionário após aceite deste termo. Foram excluídos aqueles questionários em que o participante demonstrasse interesse em desistir da participação na pesquisa, porém não houve nenhuma ocorrência deste evento.

Um estudo piloto foi realizado com 4 profissionais de Fisioterapia especialistas na área de terapia intensiva com área de atuação em Neonatologia e Pediatria, com o objetivo de refinar o instrumento de coleta, identificando questões que gerassem dificuldades de compreensão, sendo realizado adequações após essa etapa. Os questionários respondidos nessa fase não foram considerados como parte da presente amostra.

Um questionário estruturado com 19 perguntas foi elaborado para ser utilizado como instrumento nessa pesquisa. Um estudo piloto foi realizado com 4 profissionais de Fisioterapia especialistas na área de terapia intensiva com área de atuação em Neonatologia e Pediatria, com o objetivo de refinar o instrumento de coleta, identificando questões que gerassem dificuldades de compreensão, sendo realizado adequações após essa etapa. Os questionários respondidos nessa fase não foram considerados como parte da presente amostra. Foi composto na sua maioria por perguntas objetivas, porém três perguntas com o objetivo de evitar indução nas respostas dos participantes foram discursivas, mas mesmo estas tiveram suas respostas categorizadas e contabilizadas, mantendo a característica quantitativa do estudo. Além disso, buscando evitar viés de aferição, os questionários não apresentaram nenhuma identificação, garantindo o anonimato dos participantes e uma maior veracidade nas respostas.

A amostra foi de conveniência, composta pela quantidade de questionários que conseguiram ser preenchidos no período de coleta de dados descrito. As variáveis estudadas nesta pesquisa serão referentes a três domínios: o perfil do profissional, o conhecimento do profissional sobre as evidências científicas existentes acerca dos benefícios da MP em pacientes pediátricos críticos por meio de questões subjetivas com gabarito pré-determinado. $\mathrm{E}$ a percepção do participante da pesquisa sobre a importância da MP nestes pacientes além das barreiras vivenciadas.

Após a coleta dos dados, estes foram tabulados e a análise estatística descritiva foi realizada, onde foram utilizados conceitos da estatística descritiva para calcular as frequências absolutas e relativas (percentuais) das variáveis categóricas. Os dados foram tabulados em planilhas de texto do Microsoft Excel 2013, foi realizado um procedimento de conferência e limpeza dos dados para evitar possíveis inconsistências nos resultados, em seguida, foram analisados no software de análise estatística Stata, v.12.

\section{Resultados}

Dos 50 questionários respondidos na plataforma, foram consideradas 48 respostas. Durante o registro dos dados, foi identificado dois questionários exatamente iguais, desta forma, considerando um risco elevado de ter sido respondido pelos mesmos participantes, optou-se por excluí-los e evitar o risco de viés de coleta. Na tabela 1 estão apresentadas as características sociodemográficas, formação e atuação profissional, e o conhecimento relacionado à mobilização precoce do paciente crítico. Dos participantes analisados 75\% não possuem título de especialistas. Todos os profissionais disseram se atualizar nos assuntos ligados à área de pediatria, com base nos seus conhecimentos sobre mobilização precoce, relataram utilizar na sua prática clínica. Quanto aos meios que empregavam para sua atualização, 83,3\% utilizam artigos científicos e $81,2 \%$ utilizam cursos. 
Tabela 1. Características dos fisioterapeutas que atuam em UTI Pediátrica-2020 (n=48)

\begin{tabular}{|c|c|}
\hline Características & n (\%) \\
\hline \multicolumn{2}{|l|}{ Sexo } \\
\hline Feminino & $44(91,7)$ \\
\hline Masculino & $4(8,3)$ \\
\hline \multicolumn{2}{|l|}{ Tempo de graduação } \\
\hline Menos de 5 anos & $7(16,7)$ \\
\hline 5 a 10 anos & $18(37,5)$ \\
\hline Mais de 10 anos & $23(47,9)$ \\
\hline \multicolumn{2}{|l|}{ Tempo de atuação } \\
\hline Menos de 5 anos & $19(39,6)$ \\
\hline 5 a 10 anos & $20(41,7)$ \\
\hline Mais de 10 anos & $9(18,7)$ \\
\hline \multicolumn{2}{|l|}{ Especialização ${ }^{1}$} \\
\hline Não possuo & $1(2,4)$ \\
\hline Residência na área hospitalar em saúde da criança & $4(8,3)$ \\
\hline Residência na área hospitalar & $7(14,6)$ \\
\hline Residências em outras áreas & $0(0)$ \\
\hline Pós-graduação hospitalar ou em terapia intensiva pediátrica & $22(45,8)$ \\
\hline Pós-graduação hospitalar ou terapia intensiva geral & $9(18,7)$ \\
\hline Pós-graduação em pediatria & $6(12,5)$ \\
\hline Pós-graduação em outras áreas & $11(22,9)$ \\
\hline \multicolumn{2}{|l|}{ Título de especialista } \\
\hline Sim & $12(25)$ \\
\hline Não & $36(75)$ \\
\hline \multicolumn{2}{|l|}{ Procura se atualizar nos assuntos à área de pediatria? } \\
\hline Sim & $48(100)$ \\
\hline Não & $0(0)$ \\
\hline \multicolumn{2}{|l|}{ Meios que emprega para sua atualização ${ }^{1}$} \\
\hline Artigos Científicos & $40(83,3)$ \\
\hline Conversa com outros profissionais da área & $22(45,8)$ \\
\hline Cursos & $39(81,2)$ \\
\hline Especializações & $12(25)$ \\
\hline Notícias de Internet & $8(16,7)$ \\
\hline Workshops & $5(10,4)$ \\
\hline Livros & $10(20,8)$ \\
\hline
\end{tabular}

1 pergunta com opção de marcar uma ou mais opções.

Quanto à realização da mobilização precoce, $41,7 \%$ fisioterapeutas disseram que deve ser realizada nas primeiras 24 horas da admissão após estabilização hemodinâmica, e 8,3\% não sabiam opinar. Destaca-se que 4,2\% relataram que deve ser realizada independente do tempo, após admissão, desde que a criança tenha estabilidade hemodinâmica, e após a estabilização hemodinâmica do paciente, o que ocorre geralmente entre 24 e $48 \mathrm{~h}$ da admissão na UTI, Tabela 2. 
Conforme observado no Gráfico 1, os profissionais da equipe multidisciplinar de saúde (enfermeiro, farmacêutico, fisioterapeuta, fonoaudiólogo, médico, nutricionista, técnico de enfermagem, terapeuta ocupacional) participavam da prática da mobilização precoce na sua rotina assistencial, 83,3\% relataram ser fisioterapeutas.

Figura 1. Membros da equipe multidisciplinar que participam da MP na Unidade de Terapia Intensiva Pediátrica-2020

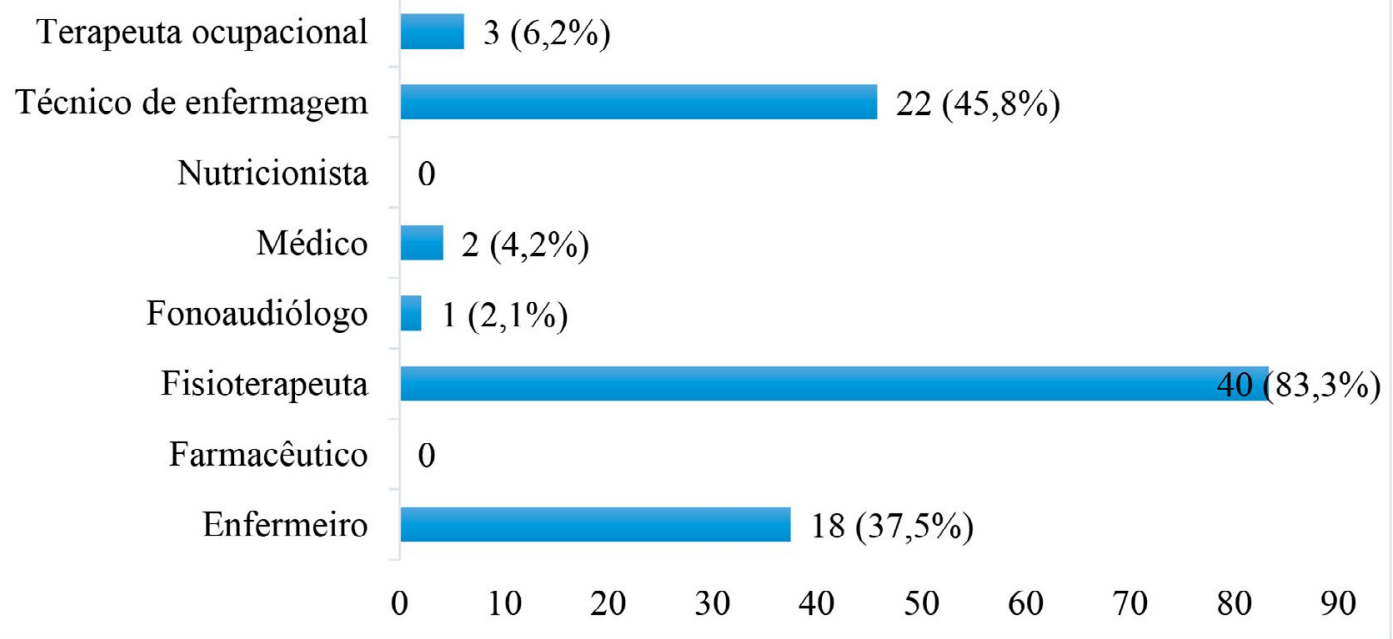

Observou-se ainda que relacionado às barreiras institucionais e da equipe, 24 profissionais (50\%) relataram falta de conhecimento da equipe, 22 profissionais $(45,8 \%)$ ausência de protocolo de mobilização precoce e 20 profissionais $(41,7 \%)$ relataram restrição de tempo e número de pacientes para atender, Gráfico 2.

Figura 2. Barreiras institucionais e da equipe da MP na Unidade de Terapia Intensiva Pediátrica-2020

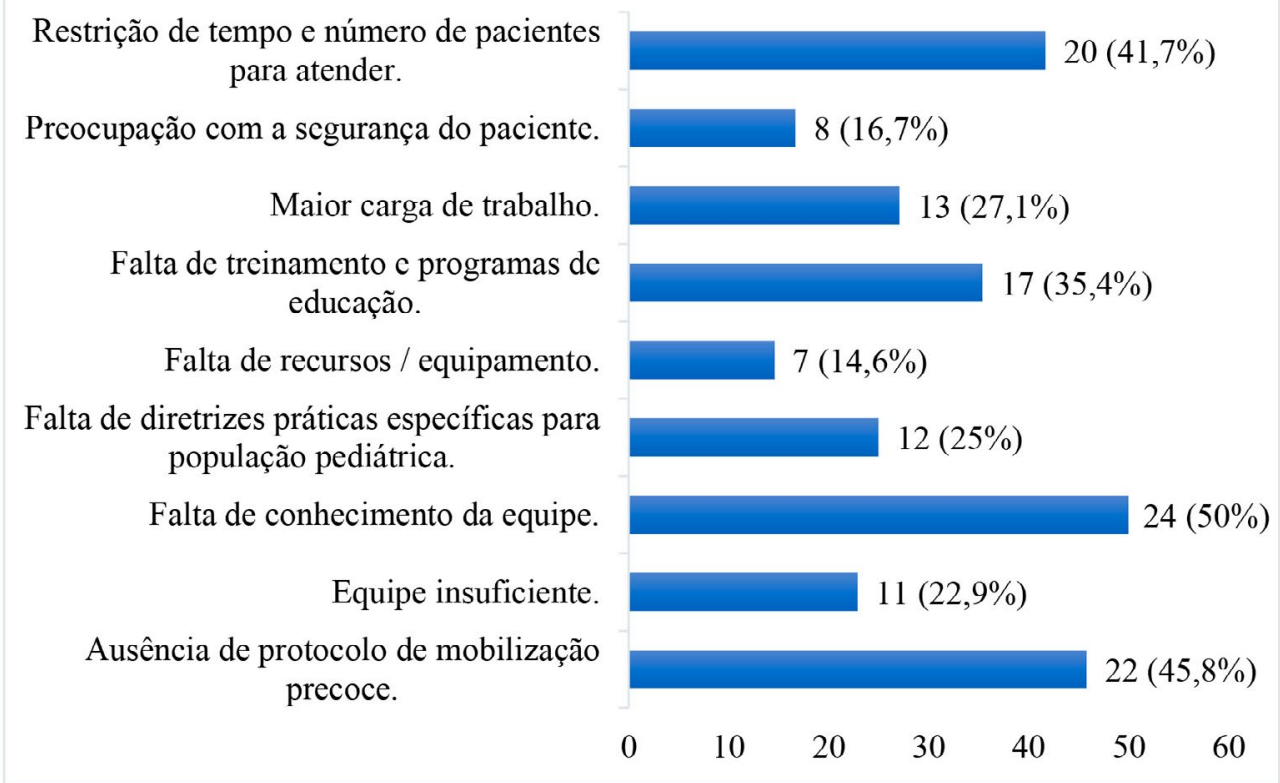

Referente às práticas da mobilização precoce em UTI pediátricas, pode-se observar que cinesioterapia (64,6\%), deambulação $(58,3 \%)$ e sedestação $(58,3 \%)$ são as principais condutas que os profissionais acreditam fazer parte da mobilização precoce. Quanto à prática de deambulação em crianças ventiladas $64,40 \%$ dos profissionais utiliza como principais critérios para realização: o nível de colaboração $(41,7 \%)$ e estabilidade hemodinâmica do paciente $(33,3 \%)$, Tabela 2. 
Condutas

$\begin{array}{lc}\text { Mudança de decúbito } & 4(8,3) \\ \text { Posicionamento } & 14(29,2) \\ \text { Mobilização Articular } & 11(22,9) \\ \text { Alongamento } & 8(16,7) \\ \text { Cinesioterapia } & 31(64,6) \\ \text { Controle de tronco e/ou cervical } & 1(2,1) \\ \text { Sedestação } & 28(58,3) \\ \text { Estímulo vestibular } & 2(4,2) \\ \text { Ortostase } & 20(41,7) \\ \text { Deambulação } & 28(58,3)\end{array}$

Deambulação com ventilação mecânica

$\begin{array}{lr}\text { Não sei opinar } & 3(6,2) \\ \text { Não } & 14(29,2) \\ \text { Sim } & 31(64,6)\end{array}$

Critérios para utilizar a deambulação em ventilação mecânica

Nível de colaboração

$20(41,7)$

Nível de sedação

Estabilidade hemodinâmica

Ausência de drogas vasoativas

Uso de traqueostomia

Segurança do paciente

$6(12,5)$

Motivo da IOT controlado

Parâmetros ventilatórios em redução

$4(8,3)$

Força muscular

Capacidade de ortostase

$5(10,4)$

Colaboração da equipe

Colaboração da família

Estrutura física

Momento em que deve ser realizada a mobilização precoce

Primeiras 24h da admissão e após estabilização hemodinâmica

Independente do tempo, durante o internamento na UTI

Não sabem opinar

Entre $1^{\circ}$ e $10^{\circ}$ dia após admissão e estabilização hemodinâmica

Primeiras 72 horas da admissão e após estabilização hemodinâmica

Outras opções

Eventos adversos

Hipotensão/Hipertensão

Queda da saturação periférica de oxigênio (SpO2)

Aumento do trabalho respiratório

Alteração do estado mental (agitação ou sonolência)

Dor ou desconforto

Perda de dispositivos (acesso, sonda, etc.)

Extubação acidental

$7(14,6)$

Queda 
Ainda sobre a rotina assistencial dos profissionais, observou-se que $31,3 \%$ utilizam escalas para acompanhamento do declínio funcional dos pacientes. Das escalas, 7 profissionais $(14,6 \%)$ citaram a Functional Status Scale (FSS) pediátrica, 1 profissional $(2,1 \%)$ Alberta, 1 profissional (2,1\%) Denver, e seis profissionais marcaram que utilizavam escalas, mas não relataram qual.

\section{Discussão}

Este estudo teve como principal objetivo avaliar o conhecimento dos fisioterapeutas que atuam em UTIP, assim como verificar a sua prática clínica, quanto à mobilização precoce em pacientes pediátricos críticos. A prática da MP vem se destacando tanto na população adulta quanto na população pediátrica. Em adultos gravemente enfermos, estudos mostram que a mobilização precoce é viável, segura, econômica e melhoram resultados funcionais de pacientes a curto e longo prazos, entretanto estudos na população pediátrica são escassos. $\frac{9.10}{10}$

Pesquisas recentes revelam que a MP em UTIP é segura e viável quando abordada sistemicamente, estão associadas a ganhos significativos em desfechos físicos e neuro cognitivos. ${ }^{9.11,12}$ Os resultados revelam que apesar de alguns profissionais relatarem que não há evidência científica que suportem a realização da MP, todos os profissionais, com bases nos seus conhecimentos, utilizam a MP em sua prática clínica.

Estudos definem mobilização precoce como qualquer terapia de mobilidade realizada dentro das 48 horas após a admissão em UTIP, contraindicada nas situações clínicas de instabilidade hemodinâmica, instabilidade respiratória, instabilidade neurológica e eventos pós cirúrgicos estão ausentes. ${ }^{2,13} \mathrm{Em}$ outros estudos, a mobilização precoce foi definida como segura e viável em 72 horas após admissão em UTIP.12,14 Nesse estudo embora $45,2 \%$ dos profissionais disseram que deve ser realizada nas primeiras 24 horas da admissão após estabilização hemodinâmica, o fato de ter tido ocorrências de variedade de tempo, demonstram na prática clínica a falta de consenso em relação quando deve ser iniciada a mobilização precoce nessa população.

O apoio e a assistência da equipe multidisciplinar são fundamentais para o sucesso do programa de MP. 10 Em um estudo realizado por Fagundes et al. ${ }^{15}$, sobre o conhecimento e percepção da equipe multiprofissional sobre mobilização precoce em UTIP de um hospital universitário, mostrou que quanto maior o conhecimento sobre MP, maior a percepção sobre a sua importância. Revelou ainda que dentro da equipe multiprofissional (fisioterapeuta, médico, enfermeiro e técnico de enfermagem) a MP é vista com maior importância pelos fisioterapeutas. Nessa pesquisa, dentre os membros da equipe multidisciplinar na prática assistencial dos participantes, os profissionais mais citados foram os fisioterapeutas, técnicos de enfermagem e enfermeiros. Vale ressaltar ainda que, alguns profissionais não citaram o fisioterapeuta como profissional que realiza o procedimento apesar da questão incluir na lista o fisioterapeuta, divergindo do resultado que todos relatarem realizar a MP em sua prática assistencial.

No estudo de Choong et al. $\frac{16}{}$ com 61 médicos e 27 fisioterapeutas atuantes em UTIP Canadenses, a falta de diretrizes foi a principal barreira institucional relatada (75,4\% médicos/ 48,1\% fisioterapeutas), também citado pelos participantes desta pesquisa, porém em uma menor frequência (26,2\%). Outro estudo realizado por Choong et al. ${ }^{13}$ demonstra que as diretrizes da prática institucional podem facilitar avaliações oportunas dos pacientes e possibilitar uma mobilização segura o mais precoce possível na maioria das crianças gravemente doentes. Em outros estudos, as principais barreiras citadas são limitações de recursos, necessidade de cooperação do paciente e apreensão com a mobilização precoce expressada por profissionais de saúde e cuidadores familiares. $\frac{12}{}$ Nessa pesquisa, as principais barreiras citadas foram a falta de conhecimento da equipe, restrição de tempo e número de pacientes para atender, bem como ausência de protocolo de mobilização precoce. O conhecimento da equipe acerca dos benefícios, da importância e da segurança através de programas educacionais e implementação de protocolos e diretrizes especificas para MP na população pediátrica são imprescindíveis para o sucesso da terapia. 
A população pediátrica representa uma população heterogênea, com estágios de desenvolvimento diferentes, com diversas habilidades cognitivas e funcionais e uma variedade de diagnósticos, gerando mais um desafio para implementação de um plano padrão de reabilitação nas UTIP, pois a capacidade de cumprir as atividades são variáveis. $910 \mathrm{Um}$ estudo de Wieczorek et al. ${ }^{17}$ definiu níveis de atividade crescentes de mobilidade com base no estado clínico do paciente, categorizando como terapias no leito ou terapias de mobilidade. As terapias no leito incluíram amplitude de movimento passiva e ativa, posicionamento ativo e passivo. As atividades de mobilidade incluíram sentar-se na beira da cama, sentar-se, transferir, deambular e brincar. Neste estudo, as principais condutas citadas foram cinesioterapia (passiva e ativa), deambulação, sedestação, que corroboram com a literatura atual. Além das práticas convencionais de mobilização, outros estudos usaram videogames/interativos e/ou cicloergômetro para facilitar a mobilização.,113 Intervenções utilizando realidade virtual e exergaming não foram citados pelos participantes deste estudo. Essas intervenções poderiam tornar o atendimento mais interativo e atrativo, visto que é uma modalidade popular entre as crianças, podendo obter melhor conformidade do que os tradicionais exercícios.?

Betters et al. avaliaram ${ }^{8}$ se era possível e seguro o uso da mobilização precoce em pacientes ventilados, realizando atividades exclusivas para a capacidade de tolerância de cada paciente, das mais simples como mudanças de posicionamento até a mais avançada como deambulação. Quando os profissionais foram questionados pela primeira vez, antes de iniciar o protocolo, sobre a segurança da prática, 30\% responderam ser seguro, já na segunda vez durante o acompanhamento, 63\% disseram ser seguro mobilizar o paciente durante a ventilação mecânica. O nível de sedação do paciente para os profissionais é considerado como uma barreira, pois o paciente tem que estar colaborativo. Outro estudo relata que pacientes entubados comumente tinham contraindicações de terapias de mobilidade. ${ }^{13}$ No presente estudo, $64,60 \%$ relataram que crianças que apresentem previamente capacidade de deambulação têm indicação de realizar no período de ventilação mecânica, tendo como principais critérios para realização o nível de colaboração do paciente e estabilidade hemodinâmica.

Os principais eventos adversos relatados pelos participantes, de acordo com sua prática assistencial, foram o aumento do trabalho respiratório, hipotensão/ hipertensão, dor ou desconforto. Outro estudo relata como barreiras eminentes, instabilidade hemodinâmica, deslocamento acidental de dispositivos, quedas, dor e ansiedade. ${ }^{10}$ Entretanto, pesquisas pediátricas de larga escala não relatam eventos adversos relacionados às práticas de mobilização precoce.,.10,17 No estudo de Choong et al., as barreiras comumente informadas pelos profissionais foram instabilidade clínica do paciente, paciente em VM, risco de deslocamento de dispositivos ou cateteres e sedação excessiva. ${ }^{\cdot \underline{16}}$

A mobilização precoce tem como um dos principais objetivos restabelecer a funcionalidade do paciente em seu estado prévio à internação. Para isso, se torna imprescindível o uso de ferramentas para mensurar a condição funcional dos pacientes, como a utilização de escalas. $\frac{18}{}$ Nesta pesquisa, $68,8 \%$ relataram não utilizar escalas na sua prática assistencial, dos participantes que utilizam escalas $14,6 \%$ citaram a FSS pediátrica. A FSS pediátrica, traduzida e validada no Brasil, é uma escala desenvolvida com base conceitual em escalas de atividades de vida diária e comportamento adaptativo, seu objetivo é avaliar os desfechos funcionais de pacientes pediátricos hospitalizados. ${ }^{19}$ As escalas são uma ferramenta essenciais para mensuração, podendo ser determinados os desfechos funcionais motores e cognitivos dos pacientes após a alta da unidade de terapia intensiva pediátrica. $\frac{18}{E}$ imprescindível que os fisioterapeutas intensivistas utilizem escalas funcionais no planejamento da MP, no momento da transferência da UTI para as unidades de internação e na alta hospitalar.

Sugere-se, assim, que sejam estimulados programas educacionais e a implementação de protocolos e diretrizes voltadas para MP na população pediátrica, assim como pesquisas em maior escala voltadas à análise da prática pelos profissionais de fisioterapia no estado da Bahia. 
Este estudo teve algumas limitações, a ferramenta através de questionário on-line traz a possibilidade de viés de aferição, uma vez que a interpretação das perguntas pode ser diferente de acordo com cada participante, mesmo tendo sido feito um estudo piloto previamente para calibração das perguntas. Finalmente, a baixa adesão dos profissionais influenciou no tamanho da amostra, interferindo na validade externa do estudo, sendo, desta forma, prudente considerar os resultados obtidos para a população estudada considerando as características assistenciais regionais.

\section{Conclusão}

Verificamos que todos os fisioterapeutas realizam a prática da MP em sua rotina assistencial nesta amostra. Observou-se ainda que mais da metade dos participantes afirmam possuir conhecimento sobre as evidências científicas acerca da MP; poucos foram os profissionais que relataram não haver evidência que suporte sua realização. Nossos achados também demonstram a falta de conhecimento da equipe multiprofissional como a principal barreira relatada pelos participantes para a prática da mobilização precoce.

\section{Contribuições dos autores}

Todos os autores do artigo tiveram igual participação na concepção e delineamento do estudo, na produção dos dados, na análise estatística, na preparação e redação do manuscrito e na revisão crítica final após a conclusão do trabalho.

\section{Conflitos de interesses}

Nenhum conflito financeiro, legal ou político envolvendo terceiros (governo, empresas e fundações privadas, etc.) foi declarado para nenhum aspecto do trabalho submetido (incluindo, mas não se limitando a subvenções e financiamentos, participação em conselho consultivo, desenho de estudo, preparação de manuscrito, análise estatística, etc.).

\section{Referências}

1. Santos LJ, Silveira FS, Müller FF, Araújo HD, Comerlato JB, Silva MC, et al. Avaliação funcional de pacientes internados na Unidade de Terapia Intensiva adulto do Hospital Universitário de Canoas. Fisioter Pesqui. 2017;24(4):437-43. https://doi.org/10.1590/18092950/17720924042017

2. Choong K, Foster G, Fraser DD, Hutchison JS, Joffe AR, Jouvet $\mathrm{PA}$, et al. Acute rehabilitation practices in critically ill children: A multicenter study. Pediatr Crit Care Med. 2014;15(6):e270-9. https://doi.org/10.1097/pcc.0000000000000160

3. Guedes LPCM, Oliveira MLC, Carvalho GA. Efeitos deletérios do tempo prolongado no leito nos sistemas corporais dos idosos - uma revisão. Rev Bras Geriatr Gerontol. 2018;21(4):499-506. https://doi.org/10.1590/1981-22562018021.170167

4. Cameron S, Ball I, Cepinskas G, Choong K, Doherty TJ, Ellis CG, et al. Early mobilization in the critical care unit: $A$ review of adult and pediatric literature. J Crit Care. 2015;30(4):664-72. http://dx.doi. org/10.1016/j.jcrc.2015.03.032

5. Abdulsatar F, Walker RG, Timmons BW, Choong K. "WiiHab" in critically ill children: A pilot trial. J Pediatr Rehabil Med. 2013;6(4):193-204. https://doi.org/10.3233/prm-130260

6. Piva TC, Ferrari RS, Schaan CW. Protocolos de mobilização precoce no paciente crítico pediátrico: revisão sistemática. Rev Bras Ter Intensiva. 2019;31(2):248-57. https://doi. org/10.5935/0103-507x.20190038

7. Joyce $C L$, Taipe C, Sobin B, Spadaro M, Gutwirth B, Elgin L, et al. Provider Beliefs Regarding Early Mobilization in the Pediatric Intensive Care Unit. J Pediatr Nurs. 2018;38:15-9. https://doi. org/10.1016/j.pedn.2017.10.003

8. Betters KA, Hebbar KB, Farthing D, Griego B, Easley T, Turman $\mathrm{H}$, et al. Development and implementation of an early mobility program for mechanically ventilated pediatric patients. J Crit Care. 2017;41:303-8. https://doi.org/10.1016/j.jcrc.2017.08.004

9. Choong K, Chacon M, Walker R, Al-Harbi S, Clark H, Al-Mahr G, et al. In-Bed Mobilization in Critically III Children: A Safety and Feasibility Trial. J Pediatr Intensive Care. 2015;4(4):225-34. https:// doi.org/10.1055/s-0035-1563545

10. Walker TC, Kudchadkar SR. Early mobilization in the pediatric intensive care unit. Transl Pediatr. 2018;7(4):308-13. https:// dx.doi.org/10.21037\%2Ftp.2018.09.02 
11. Engel HJ, Needham DM, Morris PE, Gropper MA. ICU early mobilization: From recommendation to implementation at three medical centers. Crit Care Med. 2013;41(9 Suppl.1):69-80. https:// doi.org/10.1097/ccm.0b013e3182a240d5

12. Cuello-Garcia CA, Mai SHC, Simpson R, Al-Harbi S, Choong K. Early Mobilization in Critically III Children: A Systematic Review. J Pediatr. 2018;203:25-33.e6. https://doi.org/10.1016/j. jpeds.2018.07.037

13. Choong K, Awladthani S, Khawaji A, Clark H, Borhan A, Cheng J, et al. Early Exercise in Critically III Youth and Children, a Preliminary Evaluation: The wEECYCLE Pilot Trial. Pediatr Crit Care Med. 2017;18(11):e546-54. https://doi.org/10.1097/ pcc.0000000000001329

14. Tsuboi N, Nozaki H, Ishida Y, Kanazawa I, Inamoto M, Hayashi $\mathrm{K}$, et al. Early Mobilization after Pediatric Liver Transplantation. J Pediatr Intensive Care. 2017;06(03):199-205. https://doi. org/10.1097/pcc.0000000000001815

15. Fagundes BDS, Garcia CSNB, Werner J. Mobilização precoce no paciente pediátrico criticamente enfermo: conhecimento e percepção da equipe multiprofissional de um hospital universitário. Fisioter Bras. 2019;19(6):812. https://doi. org/10.33233/fb.v19i6.2502
16. Choong K, Koo KKY, Clark H, Chu R, Thabane L, Burns KEA, et al. Early mobilization in critically III children: A survey of canadian practice. Crit Care Med. 2013;41(7):1745-53. https://doi. org/10.1097/ccm.0b013e318287f592

17. Wieczorek B, Ascenzi J, Kim Y, Lenker H, Potter C, Shata NJ, et al. PICU Up!: Impact of a Quality Improvement Intervention to Promote Early Mobilization in Critically III Children. Pediatr Crit Care Med. 2016;17(12):e559-66. https://doi.org/10.1097/ pcc.0000000000000983

18. Ferreira LL. Escalas de avaliação funcional em terapia intensiva: Revisão de literatura. Rev Aten Saúde [Internet]. 2018;16(2359-4330):108-14. Disponível em: https://seer.uscs.edu. br/index.php/revista_ciencias_saude/article/view/5191

19. Pereira GA, Schaan CW, Ferrari RS. Avaliação funcional em pacientes pediátricos após alta da unidade de terapia intensiva por meio da Functional Status Scale. Rev Bras Ter Intensiva. 2017;29(4):460-5. https://doi.org/10.5935/0103-507x.20170066 